\title{
La radio educativa como herramienta de alfabetización mediática en contextos de interculturalidad
}

\author{
Educational radio as a media literacy tool in intercultural \\ contexts
}

\author{
Carias Pérez, F., Hernando Gómez, A. y Marín-Gutiérrez, l. ${ }^{1}$ \\ Recibido: 13-05-2021 - Aceptado: 20-08-2021 \\ https://doi.org/10.26441/RC20.2-2021-A5
}

\begin{abstract}
RESUMEN: El siguiente artículo presenta un análisis sobre el uso de la radio educativa en contextos de educación intercultural y cómo, desde su puesta en práctica, se advierten elementos de la alfabetización mediática e informacional, lo cual facilita y dinamiza la construcción de una adecuada comunicación intercultural. La investigación ha sido realizada en Chile, específicamente en escuelas rurales de las comunas de San Juan de la Costa y San Pablo, con la participación de estudiantes y profesores de educación básica y media, en su mayoría de origen mapuche huilliche, pueblo originario del sur de ese país. La metodología utilizada posee un enfoque de carácter cualitativo, teniendo como estrategias de recolección de datos entrevistas semiestructuradas a informantes claves, observación participante in situ (ambas de manera presencial y virtual) y la etnografía virtual. Los resultados principales han evidenciado que la radio educativa puede generar contenidos formativos para potenciar los procesos de emancipación cultural generando, al mismo tiempo, espacios de reflexión y pensamiento crítico en los estudiantes. La alfabetización mediática e informacional resulta pertinente como herramienta pedagógica para transversalizar los procesos de enseñanza y aprendizaje en el contexto educativo chileno, ya que permite tomar elementos de los medios de comunicación para la construcción de sociedades democráticas. Y en tanto la comunicación intercultural, la capacidad de los estudiantes y profesores de crear y producir contenidos radiofónicos desde sus realidades étnicas, los cuales pueden ser difundidos y puestos al servicio de sus culturas para la promoción de sus tradiciones y formas de vida.
\end{abstract}

Palabras clave: alfabetización mediática; comunicación intercultural; radio; cultura; educación intercultural; medios de comunicación de masas; TIC.

ABSTRACT: The following article presents an analysis of the use of educational radio in intercultural education contexts and how, from its implementation, elements of media and information literacy are noticed, which facilitates and encourages the construction of adequate intercultural communication. The

\footnotetext{
${ }^{1}$ Fernando Carias Pérez es Doctorando en Comunicación por las Universidades de Huelva, Sevilla, Málaga y Cádiz en la línea de Alfabetización Mediática y Educomunicación. Magister en Ingeniería de Medios para la Educación por la Universidad de Lisboa (Portugal), Université de Poitiers (Francia) y Universidad Nacional de Educación a distancia UNED (España). Docente y Asesor curricular de la Unidad de Desarrollo Docente y Curricular en la Universidad de Los Lagos (Chile). fernando.carias@ulagos.cl, https://orcid.org/0000-0002-6955-6125

Ángel Hernando Gómez es Doctor en Psicología por la Universidad de Huelva y Profesor Titular del Departamento de Psicología Social, Evolutiva y de la Educación de la Universidad de Huelva. Es Editor Asociado de la revista Comunicar y Miembro del Grupo de Investigación «AGORA, Grupo de estudios e investigaciones educativas en tecnologías de la comunicación, Orientación e Intervención Sociocultural» dentro del Plan Andaluz de Investigación (HUM-648). angel.hernando@dpsi.uhu.es, http://orcid.org/0000-0002-6414-5415

Isidro Marín-Gutiérrez es Doctor en Antropología Social por la Universidad de Granada y Profesor del Departamento de Antropología Social de la Universidad de Sevilla. Sus líneas de investigación están centradas en la Educomunicación, nuevas tecnologías, consumo de sustancias, rituales con enteógenos y plagio. imgutierrez@us.es, https://orcid.org/0000-0002-6858-0983
} 
research has been carried out in Chile, specifically in rural schools in the communes of San Juan de la Costa and San Pablo, with the participation of students and teachers of basic and high school education, mostly of Mapuche huilliche origin, a native people of the south from that country. The methodology used has a qualitative approach, having as data collection strategies semi-structured interviews with key informants, participant observation in situ (both face-to-face and virtual) and virtual ethnography. The main results have shown that: Educational radio can generate training content to enhance the processes of cultural emancipation, generating, at the same time, spaces for reflection and critical thinking in students. Media and information literacy is pertinent as a pedagogical tool to mainstream the teaching and learning processes in the Chilean educational context, as it allows taking from the media, elements for the construction of democratic societies. And as communication intercultural, the ability of students to create and produce radio content from their own realities, which can be disseminated and put at the service of their cultures while promoting their ways of life.

Keywords: media literacy; intercultural communication; radio; culture; intercultural education; mass media; ICT.

\section{Introducción}

La radio, y en especial aquella que se enfoca en su uso desde una perspectiva educativa, ha sido desde 1920 (Grise, Epstein \& Lukin, 1974), un recurso omnipresente en los contextos pedagógicos en cualquiera de sus niveles y tipologías. Las escuelas, los centros de formación técnica y las universidades han utilizado en diferentes formatos este recurso comunicacional para mediar los procesos de enseñanza - aprendizaje. Actualmente, han ido variando las formas de difusión y los alcances de la radio y hoy no depende solamente de las ondas hertzianas. Internet y los medios digitales han reconfigurado el rol de la radio, manteniendo la esencia del lenguaje radiofónico. Este medio de comunicación ha servido para generar instancias de formación vinculadas a proyectos e iniciativas, donde además de componentes pedagógicos y comunicacionales, existen elementos asociados a la cultura y las tradiciones de los pueblos. El presente trabajo es una investigación sobre educomunicación e interculturalidad, teniendo como dínamo el uso de la radio educativa en escuelas rurales del sur de Chile, donde estudiantes y docentes producen material radiofónico para ser difundidos en emisoras comunitarias y comerciales de la provincia de Osorno, así como en las páginas web de los colegios que participan del estudio.

Por otro lado, la alfabetización mediática e informacional (AMI) es una de las herramientas más importantes para fomentar el acceso equitativo y crítico a la información y al conocimiento, como también para la promoción de medios de comunicación independientes, libres y pluralistas. Desde ella se logra reconocer el papel y la importancia de la comunicación y todo aquello que la compone y determina (medios de comunicación, información o semiótica), así como la preponderancia e impacto que estos mismos elementos mediáticos tienen en nuestra vida cotidiana. Esto nos lleva a afirmar que la alfabetización mediática e informacional viene a ofrecernos, como ciudadanos, insumos y facultades para comprender desde una perspectiva reflexiva y crítica las funciones de los medios de comunicación e información, otorgándonos la posibilidad de tomar decisiones pertinentes como usuarios y generadores (productores) de contenido mediático (Pérez Escoda, 2017).

Otro componente conceptual que atañe a este estudio es lo referido a la comunicación intercultural, un ámbito donde se interceptan las tecnologías comunicativas interactivas y las nuevas formas de experimentación tecno social, con nuevos actores, tradicionalmente discriminados e invisibilizados, que hoy hacen presencia en los medios de comunicación y operan demandando espacios multimediáticos (Pereira, 2010). Esto se evidencia en la creciente presencia de culturas indígenas en los medios de comunicación social. Resulta común leer, ver y escuchar historias de estos grupos étnicos en páginas web, redes sociales, radio, televisión o medios digitales. Estos espacios mediáticos han 
servido como catalizador y dínamo en procesos de revitalización cultural, así como promotores de las formas de vida y tradiciones de estos pueblos aborígenes. La llegada de los medios de comunicación y las Tecnologías de la Información y la Comunicación (TIC) a las comunidades indígenas, no ha sido asimilada pertinentemente por el colectivo de estos pueblos. La reflexión hacia lo interno de sus culturas carece de mecanismos idóneos para confrontar críticamente la presencia de estas tecnologías, los medios de comunicación y las mediaciones que de ellos se generan. Algunos autores como Toboso Martín (2013) afirman que la llegada de los medios de comunicación de masas y sus artefactos, las TIC, a una comunidad o institución educativa indígena, introduce nuevas formas de expresión, manifestaciones culturales foráneas, idiomas modernos dominantes, entre muchos otros elementos exógenos, los cuales provocan choques inevitables y contradicciones con las costumbres locales, pudiendo incluso, erosionar las tradiciones autóctonas. Estas mismas tecnologías también proporcionan a los pueblos tradicionales, nuevas herramientas que pueden ser utilizadas de forma creativa para preservar, fomentar y fortalecer sus propias culturas (Carias, 2018), lo cual también queda al alcance de proyectos relacionados a la alfabetización mediática y en específico a la radio educativa.

\section{Marco Referencial}

\subsection{Alfabetización mediática y su construcción conceptual}

Es desde finales del siglo XX y principios del siglo XXI que la sociedad contemporánea comienza a repensar todos los ámbitos de la interacción humana, debido a la cantidad de información que ha generado Internet. La alfabetización como término toma nuevas dimensiones y se vincula con el advenimiento de innovadoras formas de comunicación, siendo pertinente desarrollar una alfabetización al menos en tres ámbitos: digital, informacional y mediática (Pérez Escoda, 2017). Esta idea justifica la urgencia que en nuestros días supone estar alfabetizado mediáticamente, lo que también se ha denominado conciencia mediática (Pérez Tornero \& Varis, 2012), mecanismo que permitiría el acceso y la apropiación de las tecnologías, así como de los instrumentos, lenguajes y códigos que permitirían procesar la información, producirla, difundirla y la participación de los individuos, las instituciones y colectivos de toda índole. Se puede considerar, a partir de los planteamientos de Medrano, Cuervo y Martínez (2019), que las propuestas sobre alfabetización mediática son consecuencia directa de tres momentos importantes. Primero, un reconocimiento del papel de los medios de comunicación en el contexto de las sociedades democráticas y el marco internacional de los derechos humanos. Segundo, la reflexión sobre el rol de los medios de comunicación como instrumento al servicio de la educación. Y tercero, una configuración de los medios de comunicación como constructo cultural y social, donde las personas se representan, identifican y erigen.

Así, encontramos por un lado las propuestas de la UNESCO sobre alfabetización mediática e informacional, la cual supone un abordaje muy general que pone el foco en los medios de comunicación (sus contenidos generalmente), pero también en otros agentes proveedores de información como por ejemplo la misma institución escolar (Buitrago, García \& Gutiérrez, 2016), la cual utiliza la comunicación como parte de los procesos pedagógicos (Kaplún, 1998). Al mismo tiempo, se plantean desde España las ideas ofrecidas por Ferrés (2007), quien propone competencias en comunicación audiovisual, las cuales no deben desvincularse del marco AMI y pasan a denominarse ahora; competencias mediáticas, pudiendo ser digitales y/o multimodales. Con todo y las dicotomías entre lo que conceptualmente plantea la UNESCO y lo que suponen las ideas de Ferrés et al. (2007) e incluso las revisiones hechas por Pérez-Tornero y Tayie (2012), todas coinciden en el concepto de la alfabetización mediática como un conjunto de competencias relacionadas directamente a la comprensión, abordaje y análisis de los medios de comunicación. 
Se entiende que la alfabetización mediática se sitúa dentro de una serie de formas de alfabetización, que se sustentan en el desarrollo de las sociedades, siendo imperativo el avance en conjunto con la aparición de tecnologías referidas a la información y la comunicación. Los medios y la esfera comunicativa han ido cambiando apresuradamente en las dos primeras décadas del siglo XXI, lo que ha transformado también las perspectivas de la alfabetización mediática y su campo de gestión (Aguaded, Marín-Gutiérrez \& Díaz-Parejo, 2018).

Otro elemento que aplica para la comprensión y concreción de una caracterización de la alfabetización mediática tiene que ver con la alfabetización mediática como sistema de competencias. Pérez Tornero (2015) plantea las siguientes:

a. Competencias de acceso y uso.

b. Competencias relacionadas con la comprensión crítica (análisis y evaluación).

c. Competencia comunicativa y de producción creativa.

Las competencias de acceso y uso se relacionan con dos aspectos; el primero de tipo físico, referido al cómo se accede a los medios y a los contenidos mediáticos, lo que normalmente se conoce como disponibilidad. Mientras que el segundo nos remite a la capacidad cognitiva y práctica de uso de los medios de comunicación. Para ambos casos y según Pérez Tornero y Varis (2012), se involucran factores ambientales como el sistema educativo, políticas públicas, el rol de los medios, etc.

La comprensión crítica se vincula con las capacidades y habilidades de leer, comprender y evaluar los contenidos mediáticos desde una perspectiva de toma de conciencia particularmente reflexiva y con foco en la búsqueda y selección de la información a la que accedemos y sobre todo una evaluación de esa información a partir de elementos tales como el mensaje, el emisor, la veracidad o la línea editorial. Se fortalecen aspectos como el pensamiento crítico, capacidad de reflexión sobre el rol de los medios, análisis de los mensajes mediáticos desde las propias realidades y contextos.

La competencia comunicativa se genera a partir de la capacidad que tienen los individuos de crear, producir contenidos y mensajes mediáticos para difundirlos. Se involucran elementos como la creatividad, habilidades técnicas, expresivas, sociales y personales (blandas). Actualmente supone una de las competencias que se ha potenciado debido a los nuevos medios de comunicación, sobre todo aquellos que se sitúan desde una perspectiva de coparticipación y co-creación, como Instagram, Facebook, Twitter o Tik Tok. En este caso, la alfabetización mediática viene a configurarse como una herramienta vinculada al desarrollo de habilidades múltiples que van a determinar cómo entendemos, reflexionamos y nos relacionamos con los medios de comunicación (Ferrés \& Piscitelli, 2012).

\subsection{Comunicación intercultural}

Una primera aproximación a la noción de comunicación intercultural podría explicarse en base a las relaciones y valoración de la diversidad cultural desde una perspectiva comunicativa (Abdallah-pretceille, 2001). Estas relaciones pueden ser simétricas o asimétricas y además problematizantes, porque surgen de las mismas realidades multiculturales, las cuales suelen ser complejas y dinámicas desde un enfoque sociocultural (García Canclini, 2004). Los problemas más comunes de la comunicación intercultural vienen dados no solo por conflictos lingüísticos o idiomáticos, sino también por lo que Leiva Olivencia y Márquez Pérez (2012) denominan una deficiente funcionalidad comunicativa, la cual se traduce en la poca capacidad de manejar códigos distintos, en contextos diversos (familia, escuela o comunidades). Debemos tener en cuenta que hoy la comunicación, no se limita a un paradigma bimodal (emisor-receptor) de mensajes. Supone algo mucho más dinámico y revelador. 
La comunicación es una construcción de sentido, la cual puede tener diferentes niveles de significado y de lecturas que únicamente las personas con un buen conocimiento de una determinada cultura de origen pueden acceder, pues comunicación y cultura están indisolublemente interrelacionadas. La comunicación intercultural no solo se caracteriza por su función semántica, sino que también debe ser entendida desde una perspectiva de comprensión de códigos que se vinculan con distintas maneras de entender el mundo, la vida de otros y las relaciones entre las personas. El que habla, hace renacer por su discurso el acontecimiento y su experiencia del acontecimiento (Alsina, 1999). Así, la situación inherente al ejercicio del lenguaje (de comunicar), es una práctica de intercambio y diálogo trascendente. Escoffier (1991) propone cinco líneas para un adecuado y pertinente diálogo intercultural:

1. Nada es inmutable. Cuando se inicia un diálogo uno debe estar potencialmente abierto al cambio.

2. No hay posiciones universales. Todo está sujeto a crítica.

3. Hay que aprender a aceptar el conflicto y la posibilidad de que se hieran los sentimientos.

4. Hay cierta perversidad en la historia que nos han enseñado. Nuestras identidades se han hecho en oposición a la de los otros.

5. Nada está cerrado. Cualquier cuestión puede siempre reabrirse.

La comunicación intercultural no es un estilo comunicativo específico de tipo lingüístico, tal cual lo señala Pareja de Vicente, Leiva Olivencia y Matas Terrón (2020), al afirmar que es una cualidad compleja que se vincula con la predisposición, la receptividad y el talante propositivo en materia de interacción cultural, lo que la relaciona con la interculturalidad y sus complejos procesos. La comunicación intercultural es un proyecto de convivencia, de relación, comunicación y aprendizaje entre personas de distintas identidades culturales y raciales, que comparten sus conocimientos, sus formas de vida, valores y tradiciones en espacios comunes. Igualmente es un lugar para saber y conocer del "otro", mediante conexiones simétricas y de respeto que permitan espacios dialógicos y verdaderamente democráticos.

Dentro del ámbito escolar la comunicación intercultural es un elemento de importancia. Ésta se asocia con temas que promueven la inclusión en la escuela a partir de la diferencia cultural, por lo que se operativiza desde elementos relacionados con el paradigma de la educación intercultural. La comunicación intercultural implica todo un conjunto de variables básicas necesarias para facilitar la mejora de la convivencia escolar. Por ejemplo, se trata de fomentar en el contexto educativo una serie de habilidades para la escucha, la comprensión y la potenciación de la comunicación interpersonal (Leiva Olivencia \& Márquez Pérez, 2013).

Otra perspectiva que consideramos importante en tanto la comunicación intercultural está vinculada y emana de elementos y fenómenos de carácter sociocultural, socioeconómicos y sociopolíticos. Un ejemplo preciso de esto, suponen los procesos migratorios transnacionales que a gran escala han acontecido en las últimas décadas. Estas movilizaciones, generadas por factores de toda índole, se caracterizan por un "choque" de culturas que hacen inevitable la dialogicidad y el entendimiento del otro. Esto ha significado una reconfiguración de las identidades en todos los ámbitos y va generando espacios de confluencia que permiten una comunicación ente diferentes (Hadriel y Cogo, 2020).

\subsection{Sobre la radio y su rol educativo.}

La radio ha significado un importante aporte de cara al ámbito educativo. Históricamente se ha vinculado a distintas metodologías de estudio, siendo las primeras, aquellas relacionadas a los cursos 
por correspondencia, que facilitaban el acceso a la educación de los sectores más vulnerables (Grise, Epstein \& Lukin, 1974). En este sentido, Arteaga (2004), afirma que en gran medida los inicios de la radio educativa se justifican a partir de los altos índices de deserción escolar, debido a las dificultades de acceso a las escuelas y a las grandes distancias entre las poblaciones y los recintos educativos, siendo entonces una alternativa para proseguir los estudios. Al igual que la televisión, ha logrado en los procesos de enseñanza - aprendizaje una alta efectividad a través de didácticas específicas que han potenciado su uso como herramienta formativa (Araya-Rivera, 2017). El sonido, la música, los silencios y la palabra configuran lo que se conoce como el lenguaje radiofónico (Méndiz Rojas, 2004), el cual es un elemento fundamental a la hora de generar contenidos educativos con carácter formativo. Así, Merayo (2000) ofrece claves, retos y perspectivas que pueden fortalecer el uso de la radio en el contexto educativo, por ejemplo: el aprovechamiento de las nuevas y emergentes tecnologías que permitan la reconfiguración de la radio como medio de comunicación. La posibilidad de conjugarse con otros tipos de medios y así potenciar su alcance. Experimentar e innovar a través de nuevos formatos y formas de comunicación, vinculándose con diferentes actores de la sociedad, dando respuesta a las diferencias culturales, religiosas o políticas, y replanteando una "regeneración" de la radio educativa a partir de la creatividad y sus propios recursos expresivos.

Desde otra perspectiva, Perona y Veloso (2007), así como Rodero-Antón (2008), advierten que la radio educativa puede hoy, articular otros propósitos además de los ya históricamente mencionados, por ejemplo, desde la radio se puede potenciar la expresión oral y escrita de los estudiantes, la creatividad y además ampliar el conocimiento en aspectos básicos de la cultura y el conocimiento en general en todas sus instancias (arte, deportes, historia, geografía, política, ciencias, etc.) Igualmente, se fortalecen aquellas competencias de formación integral, conocidas también como competencias blandas, como lo son el trabajo en equipo, la solidaridad, la convivencia e incluso, la forma de comunicarnos y aprender.

En la actualidad, comienzan a aparecer distintas formas de hacer y entender la radio, como la ciber radio, la radio digital y el podcat (González Conde, 2014), las cuales ofrecen otra dimensión en tanto su uso educativo, pues al mismo tiempo se involucran en aspectos relacionados a la cultura y a los medios digitales, significando una alternativa atractiva para las nuevas generaciones de estudiantes, que han nacido y son usuarios de estos mismos medios. Justamente el podcast, supone una herramienta significativa desde el punto de vista educativo que permite potenciar proyectos e iniciativas formativas de distinto índole y alcance, sobre todo en contextos de educación rural, como lo evidencia el artículo de Castillo Villalobos, Monroy y Tíjaro (2012) sobre podcat y radio escolar a raíz de un proyecto desarrollado en Neusa, Cundinamarca, Colombia. Este formato consiste en un archivo de audio, que puede ser MP3, MP4 WAV, WAVE, entre otros, el cual es compartido a través de distintos medios y multiplataformas como páginas web, repositorios digitales, App de audio y música, WhatsApp y un $\sin$ fin de lugares que permiten acceder a este tipo de archivos y la información que ofrecen. En tanto su estructura, el podcat puede apoyarse de la producción de cualquiera de los formatos radiofónicos, teniendo la ventaja de ser reutilizable, es decir, permite escuchar los audios cuantas veces se desee y en cualquier lugar (Buckingham, 2019). Facilita entonces, generar contenidos de interés que los estudiantes pueden revisar y también en los cuales los docentes pueden apoyarse para generar sus clases y complementar información referidas a sus áreas disciplinares (Gutiérrez Porlán y Rodríguez Cifuentes, 2010). Una de las ventajas del podcat es que no requiere de complejos equipos ni elementos técnicos para su producción, siendo que hoy en día con un simple Smartphone y alguna App de grabación y sonido, puede realizarse (Martínez y Sánchez Vera, 2010).

Para Muñoz (1994) la radio educativa, además de vincularse al hecho pedagógico desde didácticas que utilizan la lógica radiofónica para mejorar los procesos de enseñanza-aprendizaje (Sevillano García, 2011), también puede promover capacidades para originar actitudes colectivas y positivas en los individuos como: aminorar las consecuencias de la desinformación y el uso "sin sentido" de 
los medios de comunicación. Dar indicios sobre las transformaciones tecnológicas, económicas, comunicacionales y de la ciencia en general. Y, finalmente, ofrecer el conocimiento entre los pueblos y las culturas a través de un diálogo permanente como fuente de solidaridad. A pesar de que existe un vicio histórico que ha profetizado la muerte de la radio, todavía hoy sigue siendo un medio de comunicación que se erige firme y fuerte a pesar de los vaticinios de su extinción (Consani, 2018).

En tanto la alfabetización mediática, la radio educativa juega un papel fundamental en la narrativa de este trabajo pues, mediatiza los procesos que dan respuesta a las inquietudes investigativas aquí expuestas. Al referirnos a la radio, en el contexto de presente estudio, lo hacemos como el elemento dinamizador de donde emanan los fenómenos que iremos a analizar. Trabajamos desde iniciativas pedagógicas de tres escuelas rurales al sur de Chile que utilizan la radio como herramienta para potenciar los procesos de enseñanza-aprendizaje de sus estudiantes de educación básica y media, con edades que oscilan entre los 6 y los 12 años, declarados en vínculo con la cultura mapuche-huilliche, pueblo representativo de la Patagonia chilena.

\subsection{El pueblo Mapuche y el mapuche huilliche del sur de Chile.}

Los mapuches son el grupo étnico mayoritario del país-nación que hoy se conoce como Chile. Su presencia en territorio austral es ancestral y ha logrado, a pesar de enormes dificultades y luchas, preservar elementos esenciales de su cultura hasta nuestros días. Algunos datos del último censo realizado en Chile en 2017 señalan que existen al menos un millón de personas que han declarado ser mapuches. Geográficamente se ubican a lo largo del país, siendo la octava y décima región, donde se evidencia mayor presencia de los miembros de esta cultura. La denominación huilliche se refiere a los mapuches del sur de Chile, específicamente aquellos que se encuentran en la región de Los Lagos. Según datos del mismo censo del 2017, el número aproximado en esta zona es de 50.000 miembros declarados de este grupo étnico, siendo que la mayoría se encuentran asentados en comunidades rurales y apartadas de los centros urbanos, manteniendo en muchos casos, sus formas tradicionales de vida. El Estado chileno, a través de Ministerio de Educación, ha insertado en las escuelas, programas para preservar la cultura mapuche, haciendo énfasis en el rescate del idioma propio, en el caso específico de lo mapuche huilliche, el chesungun, una de las variantes fonéticas del mapudungun. Las escuelas ubicadas al sur del país poseen la ventaja de vincularse con el contexto, tomando elementos de las tradiciones, la cultura y las formas de vida de los lugares y sus habitantes, lo cual repercute en las experiencias formativas de los estudiantes.

\section{Metodología y procedimientos.}

El plan metodológico está enmarcado en una perspectiva de carácter cualitativa a partir de un estudio de caso que acopia datos y representaciones de tres escuelas. El estudio de caso debe entenderse como un proceso de exploración caracterizado por el examen sistemático y en profundidad de casos de un fenómeno, los cuales pueden ser entidades sociales o entidades educativas únicas (Sabariego, Dorio \& Massot, 2019). Es un estudio que posee la característica de ser exploratorio y descriptivo, ya que se describen situaciones, eventos, procesos pedagógicos interactivos y experiencias únicas desde perspectivas étnicas multisituadas (Tuhiwai Smith, 2016), explorando junto con diferentes actores: docentes, investigadores, actores sociales, comunidad y especialistas temáticos y los estudiantes de las escuelas, las posibilidades socio-pedagógicas de la radio educativa como herramienta de revitalización cultural a partir de prácticas educomunicativas en contextos de interculturalidad. El diseño de esta investigación es de campo.

El enfoque cualitativo asumido en este estudio se hizo operativo recurriendo a las siguientes estrategias y técnicas de recolección de datos: 1) La perspectiva del autor, evidenciada mediante la observación participante, 2) entrevistas abiertas o semiestructuradas a informantes y participantes 
claves (profesores y profesoras, estudiantes, personal administrativo y de gestión educativa, investigadores, miembros de la comunidad de Bahía Mansa, San Juan de la Costa y Quilacahuin, investigadores) y 3) la etnografía virtual o etnografía en línea para el análisis de material digital radiofónico producido por docentes y estudiantes.

\subsection{El contexto y los datos}

La investigación se ha realizado en tres escuelas de la provincia de Osorno, al sur de Chile. Dos de ellas ubicadas en las comunas de San Juan de la Costa (Escuela Rural de Bahía Mansa y Colegio Misión San Juan) y la otra en la comuna de San Pablo (Colegio Quilacahuin). La característica principal de estos establecimientos es que se ubican en zonas rurales, apartadas considerablemente de los grandes centros urbanos siendo, además, que sus estudiantes en al menos un $85 \%$, declaran pertenecer al pueblo originario mapuche huilliche, grupo étnico característico del sur de Chile. Tienen importancia para este estudio los elementos antes descritos pues, el contexto aporta insumos de relevancia propuestos como parte de la investigación, a saber; la interculturalidad, la educación rural, la cultura mapuche huilliche, la radio como elemento educativo, dada la tradición histórica de la comuna de Osorno a partir de la experiencia de la Fundación Radio Escuela para Desarrollo Rural (FREDER).

La Escuela Rural de Bahía Mansa atendió aproximadamente 162 estudiantes durante el año 2019, momento de la investigación, trabajando únicamente con los niveles de pre-básico y básico, es decir, estudiantes entre los 5 y los 11 años de edad. Esta escuela aborda el tema de la radio desde talleres que se ofrecen como actividad complementaria formativa, lo que significa que la educación en radio no es parte del plan de estudios formal.

Estos talleres son optativos y solo asisten aquellos estudiantes interesados en participar de la radio, aprovechando que dentro de la institución existe una radio comunitaria llamada Lafkenray, la cual tiene cobertura limitada en la zona de Bahía Mansa, Pucatrihue y Mailcopue, poblados que componen parte de la comuna de San Juan de la Costa. A los talleres asisten cerca de 20 estudiantes que pueden ir rotando a lo largo del año escolar que en Chile es, generalmente, de marzo a diciembre. El número total de estudiantes que participó durante el año 2019 fue de 45, lo que supone un 27,7\% del total de alumnos de la escuela. Para el año 2020, debido a la pandemia, no se realizaron talleres pues el plantel, como todo el sistema educativo chileno, detuvo la modalidad presencial y además, la emisora cesó sus transmisiones por problemas técnicos, los cuales fueron solventados en marzo de 2021.

Para recolectar información de la Escuela Rural de Bahía Mansa se entrevistaron 8 docentes de asignaturas generales: matemáticas, lenguaje, historia y artes, además de la radio controladora y encargada de la radio y los talleres. La institución cuenta con 19 docentes. Por otro lado, se entrevistó a personal de gestión educativa de la municipalidad de San Juan de la Costa (2 personas) y una investigadora quien realizó su tesis de maestría sobre la radio de la escuela. Así mismo, se hicieron entrevistas a 20 estudiantes, más la observación participante, de la cual se recogieron datos en las visitas a terreno.

Con respecto a los colegios Misión San Juan y Quilacahuin, ambos pertenecen a la Fundación Misiones de la Costa, institución histórica en el contexto educativo de la provincia de Osorno, fundada por misioneros capuchinos holandeses en la década de los 60, de la cual forma parte también Radio La Voz de la Costa, emisora de la zona con una basta experiencia en educación radiofónica. Estos establecimientos formaron parte de la investigación durante el año 2020, ya que utilizaron el recurso radio para sortear los problemas de continuidad en sus planes de estudio, debido a las dificultades de conectividad de los estudiantes, quienes poseen poco o ningún acceso a Internet, problema común en las zonas rurales y apartadas del sur de Chile. 
El colegio Misión San Juan cuenta con una matrícula de 232 estudiantes y el colegio Quilacahuin con 144 estudiantes. De estas experiencias entrevistamos solo a los docentes encargados de crear y producir el material radiofónico que sirvió como apoyo formativo en la no presencialidad ante la emergencia sanitaria por coronavirus. En total, se realizaron 7 entrevistas a profesores de asignaturas generales de los dos colegios y una entrevista al coordinador académico de ambas instituciones, quien además, es el impulsor y encargado del proyecto que utiliza la radio como recurso educativo. Igualmente revisamos 17 archivos de audio en formato de cápsula radial, producto del material radiofónico generado por los docentes. El análisis se centró en la estructura del guion, la pertinencia disciplinar de la información, los recursos sonoros, duración y creatividad de las propuestas.

La observación participante se realizó desde dos perspectivas, la primera en el año 2019 permitió visitar una vez a la semana la escuela rural de Bahía Mansa e interactuar en los talleres de radio ofrecidos a estudiantes de básica y media, junto con la monitor responsable de la gestión de la radio. Estas dinámicas no eran pasivas, pues realizamos acompañamiento en la creación de guiones, grabaciones y programas en vivo en la radio comunitaria que existe dentro de la institución.

La otra instancia supuso una observación en el marco de la etnografía virtual, lo cual implicó visitar sitios Web de las escuelas y escuchar los materiales producidos por los estudiantes para su posterior análisis, así como acompañar en algunos momentos, a los docentes en la realización de pequeños talleres para la producción de contenidos radiofónicos. De esta última experiencia surgieron manuales para la producción de cápsulas radiales, los cuales sirvieron de apoyo a los docentes para aproximarse a la producción de radio con fines educativos.

Vale mencionar que las iniciativas de hacer radio en las tres escuelas participantes de la investigación, forman parte de proyectos institucionales que transversalizan los planes de estudios y solo en el caso de la escuela de Bahía Mansa, los programas producidos por los estudiantes entran dentro de la parrilla de programación de la radio comunitaria Lafkenray. En el caso de las escuelas Misión San Juan y Quilacahuin, las producciones son transmitidas con frecuencia diaria de lunes a viernes por Radio La Voz de la Costa y además subidas a la página web de ambas instituciones, donde los apoderados y los estudiantes acceden a los contenidos.

\subsection{Fases para el análisis}

En primera instancia se compiló toda la información recolectada previamente y se ordenó con criterio de dar respuesta y en vínculo con los objetivos planteados por la investigación. Se estableció una secuencia en el orden de los datos para facilitar el manejo de la información, siendo previamente necesario transcribir en formato digital todas aquellas entrevistas y grabaciones de audio (material multimedia) que sirvieron como insumo informativo. Se filtraron y agruparon las ideas más significativas y representativas de la línea temática y argumentativa de la tesis y posteriormente se presentaron a la herramienta de software utilizada para apoyar la concreción del proceso.

El análisis de los datos per se, se realizó a través de Atlas.ti, versión 9, a partir de unidades de análisis y categorías, ambas previamente identificadas y vinculadas al marco referencial de competencias que brinda la alfabetización mediática (competencias de acceso y uso, comprensión crítica y competencia comunicativa). Se compuso una instancia de análisis a partir de elementos de la comunicación intercultural, generando unidades de análisis y categorías que posteriormente fueron contrastadas y cruzadas para construir la trama argumentativa de los resultados y las conclusiones que ofrecemos en este trabajo.

A continuación, las unidades de análisis y sus categorías, según los ámbitos de este estudio: Alfabetización mediática y comunicación intercultural. 
Tabla 1. Unidades de análisis y categorías para el análisis de la alfabetización mediática.

\section{ALFABETIZACIÓN MEDIÁTICA}

\begin{tabular}{|l|l|}
\hline \multicolumn{1}{|c|}{ Unidad de análisis } & \multicolumn{1}{c|}{ Categorías } \\
\hline Competencias de acceso y uso. & $\begin{array}{l}\text { - Acceso a los medios de comunicación. } \\
\text { - Capacidad cognitiva en el uso de los medios de comunicación. } \\
\text { - Frecuencia de uso. }\end{array}$ \\
\hline - Uso instrumental de las TIC. \\
\hline Competencias de comprensión crítica. & $\begin{array}{l}\text { - Conciencia reflexiva sobre los medios de comunicación. } \\
\text { - Pensamiento crítico. } \\
\text { - Capacidad de análisis de los mensajes. }\end{array}$ \\
\hline Competencia comunicativa. & $\begin{array}{l}\text { - Formas de generar contenidos. } \\
\text { - Estrategias de difusión mediática. }\end{array}$ \\
\hline
\end{tabular}

Fuente: Elaboración propia.

\subsection{Unidades de Análisis y sus categorías.}

1. Competencia de acceso y uso: Se refiere a la forma que los estudiantes se vinculan y conectan con los medios de comunicación y las tecnologías de la información y la comunicación.

\section{Categorías}

1.1. Acceso a los medios de comunicación: Cómo acceden los estudiantes a los medios de comunicación, cuáles y a través de qué tipo de herramientas tecnológicas lo hacen.

1.2. Capacidad cognitiva en el uso de los medios de comunicación: Define los procesos y las habilidades cognitivas que poseen los estudiantes en tanto el uso de los medios de comunicación. Implica la lógica de uso y la forma de resolver problemas.

1.3. Frecuencia de uso: Cada cuánto tiempo acceden a los medios de comunicación y en especial las veces en que usan la radio en cualquier situación o contexto.

1.4. Uso instrumental de las TIC: Observa la lógica de uso de ciertos dispositivos como Smartphone, computadores, software y App de grabación y edición de audio. Además, toma en cuenta la capacidad de generar productos sonoros radiales a partir del uso de tecnologías.

2. Competencia de comprensión crítica: Establece mecanismos para un análisis reflexivo y crítico de los medios de comunicación a través de mecanismos de discernimiento y valoración como usuario y creador de contenidos.

\section{Categorías}

2.1. Conciencia reflexiva sobre los medios de comunicación: Los estudiantes y los docentes problematizan sobre el rol de los medios de comunicación en sus vidas y realidades cotidianas.

2.2. Capacidad de análisis de los mensajes: Poseen herramientas, tanto estudiantes como docentes, para disgregar los mensajes mediáticos, pudiendo identificar, evaluar, clasificar e interpretarlos.

2.3. Pensamiento crítico: Procesan y razonan para una toma de decisiones pertinente con respecto 
a los medios de comunicación, comunicando y retroalimentando sus ideas constantemente.

3. Competencia comunicativa: Formas y maneras de interactuar con y desde los medios de comunicación a partir de la producción de contenidos, uso de tecnologías, trabajo en equipo y maneras de comunicar sus mensajes.

\section{Categorías}

3.1. Formas de generar contenidos: Plantea la manera y lógica en que el estudiante afronta el proceso creativo en la generación de formatos radiofónicos y/o contenidos mediáticos. Evalúa el paso a paso del proceso.

3.2. Estrategias de difusión mediática: Cómo se exponen y difunden los contenidos producidos y aquellas habilidades para hacer de fácil acceso los materiales radiofónicos producidos. Supone igualmente la pertinencia del lugar donde se comparten los materiales y formatos radiofónicos.

3.3. Trabajo colaborativo: Evidencia la capacidad del trabajo entre pares para la producción de material radiofónico, así como la capacidad de interactuar entre docentes y estudiantes con el mismo fin.

Tabla 2. Unidades de análisis y categorías para el análisis de la comunicación intercultural.

COMUNICACIÓN INTERCULTURAL
\begin{tabular}{|l|l|}
\hline \multicolumn{1}{|c|}{ Unidad de Análisis } & \multicolumn{1}{c|}{ Categorías } \\
\hline Funcionalidad comunicativa. & $\begin{array}{l}\text { - Contexto del mensaje. } \\
\text { - Coherencia discursiva del mensaje. } \\
\text { - Pertinencia didáctica del mensaje. }\end{array}$ \\
\hline Códigos comunicativos. & $\begin{array}{l}\text { - Tipo de léxico. } \\
\text { - Idioma (castellano o chesungun). } \\
\text { - Lenguaje radiofónico. }\end{array}$ \\
\hline Interacción intercultural. & $\begin{array}{l}\text { - Relaciones simétricas. } \\
\text { - Capacidad de comprender al otro. } \\
\text { - Diversidad cultural. }\end{array}$ \\
\hline
\end{tabular}

Fuente: Elaboración propia.

\subsection{Unidades de análisis y sus categorías.}

1. Funcionalidad comunicativa: Determina si el mensaje de las producciones radiofónicas cumple los objetivos de ser pertinentemente adecuados desde una perspectiva intercultural. Se valoran elementos como el contexto, la otredad, valores, tradiciones e incluso su adecuado uso desde aspectos educativos y pedagógicos.

\section{Categorías}

1.1. Contexto del mensaje: Lo que se expresa corresponde y se adhiere a situaciones de carácter intercultural que valoran un diálogo respetuoso y equilibrado entre quienes se comunican.

1.2. Coherencia discursiva del mensaje: Representa la articulación de varios elementos dentro del proceso de creación de producciones radiofónicas, para darle sentido a las ideas que se desean expresar a través de la radio o en las relaciones entre estudiantes y docentes. 
1.3. Pertinencia didáctica del mensaje: Las ideas que se generan a través de la producción radiofónica, poseen elementos que facilitan los procesos formativos de los estudiantes transversalmente.

2. Códigos comunicativos: Se evidencian elementos de las culturas que están en diálogo, haciendo la comunicación intercultural, por ejemplo; diferentes formas de expresión como música, tradiciones orales, idiomas, códigos propios de cada cultura.

\section{Categorías}

2.1. Tipo de léxico: El vocabulario utilizado es pertinente y promueve la comunicación intercultural en tanto es adecuado y respeta las diferencias.

2.2. Idioma (castellano o chesungun): Cuál idioma utiliza el estudiante y los docentes para expresar sus ideas, tanto en las situaciones pedagógicas, como en la producción radiofónica.

2.3. Lenguaje radiofónico: Utilizan en la producción de formatos radiofónicos música, efectos, silencios, buena dicción y coherencia en las ideas.

3. Interacción intercultural: Resguarda elementos relacionales entre las culturas que dialogan, instancias y espacios básicos para la convivencia. Se reconoce el valor del otro y se entiende la riqueza de la diversidad entre los seres vivos.

\section{Categorías}

3.1. Relaciones simétricas: Define la idea de una condición de igualdad entre culturas y cómo es entendido y tratado el tema en las producciones radiofónicas en ese sentido.

3.2. Capacidad de comprender al otro: Se centra en el ejercicio del relativismo cultural, ponerse en el lugar del semejante y tratar de comprender sus perspectivas y formas de entender el mundo y las realidades. Aplica para estudiantes y docentes.

3.3 Diversidad cultural: Enfatiza en el entendimiento que tienen los estudiantes que producen material radiofónico, sobre la multiplicidad de formas en las que se expresan las sociedades y las culturas, generando una impronta sobre esto en sus propuestas.

Los esquemas y descripción de las unidades de análisis y las categorías aquí presentadas, dan respuesta a la información que hemos extraído y recolectado de los datos. El siguiente momento supone una etapa clave y se refiere al análisis de resultados, el cual debe ser entendido como el flujo y conexión interactiva de inferencias en procura de generar indicios resultados y conclusiones (McMillan y Schumacher, 2005).

\section{Resultados}

Partiremos y argumentaremos los resultados desde el contexto de las interacciones realizadas entre estudiantes y profesores de las escuelas participantes de la investigación, así como desde los talleres formativos, teniendo como marco el momento en que se trabaja desde la especificidad y actividades referidas a la radio como herramienta educativa. Pondremos el foco en la producción de formatos radiofónicos y el proceso manifiesto de hacer radio en su más amplio sentido, incluida la difusión de los contenidos producidos (Cápsulas informativas, micro documentales sobre diversos temas y tópicos y noticieros escolares), así como su impacto socioeducativo en el 
territorio, con especial énfasis en elementos de índole cultural, lo mapuche-huilliche (González Castro, 2014).

1. En tanto la alfabetización mediática se evidenciaron a través de las distintas categorías, elementos que piensan prácticas relacionadas a este campo. No existe un conocimiento formal con respecto a las habilidades competenciales de manera general, sin embargo, es axiomática la presencia de prácticas mediáticas (creación y producción) en "clave de alfabetización". Esto quiere decir que los docentes y los estudiantes problematizan en torno a los medios de comunicación y además producen contenidos mediáticos generando discusiones y debates sobre el rol de la radio principalmente, aunque en algunas ocasiones se planteó el tema de otros tipos de medios como la televisión y los medios digitales. Se observó en algunas sesiones de trabajo presenciales en la Escuela Rural de Bahía Mansa, la inquietud por parte de los estudiantes de asumir un papel más activo con respecto a su relación con los medios, sobre todo en el uso de aplicaciones como Instagram, Tik Tok, etc. Esto evidencia ciertas competencias de acceso y uso a los medios de comunicación.

2. Sobre la competencia de acceso y uso, se evidenció que los estudiantes y los profesores operan de manera instrumental tecnologías de la información y la comunicación como Smartphones, computadores, televisión, radio e Internet. Se demostró una alta capacidad de usabilidad con respecto a ciertos medios de comunicación, al menos desde un nivel básico y medio. Los docentes saben y están conscientes de las posibilidades que tienen los medios de comunicación para potenciar instancias de formación de sus estudiantes, sobre todo en lo referente a la lectoescritura (Braslavsky, 2013), la historia y la cultura en términos de interculturalidad (cultura nacional y cultura indígena). Los estudiantes dejaron en evidencia mediante las entrevistas, que medios como la radio, les puede ayudar a la comprensión de contenidos y áreas temáticas vinculadas con sus estudios (Labio-Bernal, Romero-Domínguez, García Orta, y García-Prieto, 2020), pero sobre todo como herramienta para conocer una parte de su esencia cultural mapuche-huilliche (Carias Pérez, Hernando Gómez \& Marín-Gutiérrez, 2021), a pesar de estar inmersos en un contexto de ruralidad, tantos los estudiantes como los docentes tienen acceso a los medios de comunicación, siendo Internet el que presenta mayor dificultad debido a lo apartado de ciertos territorios, lo que igual no interfiere en el uso y consumo consecuente y cotidiano de las TIC y los mismos medios de comunicación, pero sí evidencia elementos de brecha digital (Escuder, 2019). Un dato importante es que muchos de los docentes entrevistados estaban al tanto de los recursos formativos de la radio, sin embargo, por desconocimiento o sencillamente por desinterés, no se animaban a producir contenidos para el apoyo de sus estudiantes.

3. Con respecto a la competencia de comprensión crítica, ésta es la que se presenta menos desarrollada. A partir de la información recolectada en las entrevistas y la observación en campo, no se evidenció elementos que indicaran un posicionamiento crítico o reflexivo con respecto a los medios de comunicación. Sin embargo, eventualmente durante los ejercicios de realización de guiones o trabajos escritos, sobre temas relacionados a la cultura mapuche-huilliche en la Escuela Rural de Bahía Mansa, aparecieron ideas y acotaciones desde los docentes, los estudiantes y las autoridades de las escuelas (administrativos, dirigencia, educadores tradicionales ${ }^{2}$ ), sobre la necesidad de crear líneas temáticas que apunten al resguardo y revitalización cultural a partir del trabajo con la radio. Esto último podría asumirse como una postura que

\footnotetext{
${ }^{2}$ Los educadores tradicionales son dentro del contexto educativo chileno quienes se encargan de guiar la formación en temas vinculados a los pueblos originarios, sobre todo lengua, tradiciones, historia y cosmovisión. Su misión es de mediador intercultural y tienen la capacidad de generar didácticas con pertinencia cultural, que permitan a los estudiantes, independientemente de su origen cultural, relacionarse con el mundo indígena.
} 
repiensa la oportunidad de los medios y las tecnologías de la información y la comunicación en beneficio de salvaguardar el legado cultural del pueblo mapuche-huilliche y se relaciona directamente con la categoría de capacidad de análisis de los mensajes. Igualmente, en las entrevistas con los profesores de los colegios Misión San Juan y Quilacahuin, apareció en las conversaciones la necesidad de replantear los significados de los medios de comunicación en función de que los estudiantes sean conscientes de los efectos negativos que estos pueden tener en temas como valores ciudadanos, formas de vida y relaciones sociales.

4. La competencia comunicativa es la más desarrollada por los estudiantes, evidenciado en aquellas habilidades relacionadas a las formas de generar contenidos, uso instrumental de las TIC, estrategias de difusión mediática y trabajo colaborativo. Los estudiantes por un elemento de carácter generacional y vivencial poseen herramientas cognitivas que ponen al servicio de la producción de contenidos (Buckingham, 2003), a través de alguna app para la edición de multimedia o sencillamente mediante algún aplicativo insertado en redes sociales o incluso dentro de los dispositivos que habitualmente utilizan, tales como: Smartphone o Tablet (Soep, 2012). La idea de hacer radio desde una cabina o un estudio se traslada a la cotidianidad y los aspectos técnicos de la edición y el montaje de sonidos, efectos, silencios, entre otros, pueden ser elaborados de manera cuasi artesanal con un nivel de calidad bastante aceptable. Vale la pena señalar que el aprendizaje del uso de estos dispositivos y herramientas por parte de los estudiantes ha sido de forma autodidacta y no existe o al menos no se evidenció, ninguna sistematización de los procedimientos para la producción. Es decir, no hay un método en acuerdo común en cómo y de qué manera producir formatos radiofónicos. Resultó particularmente interesante ver a los estudiantes innovando y buscando formas de expresarse a través de la creación de contenidos radiofónicos, lo que además sirvió para registrar estrategias, desde nuestra perspectiva investigativa, que pueden ser replicadas en otros proyectos o instancias similares. Por el lado de los profesores el tema es diametralmente opuesto, pues muchos de ellos no poseen competencias en el uso de ciertas tecnologías y buscan eventualmente apoyo en los estudiantes para generar contenidos radiales. Sin embargo, algunos, han descubierto en la radio una línea de trabajo que los vincula con la innovación de sus prácticas pedagógicas (Moreno-Correa, 2020). Surgió en las entrevistas anécdotas de los profesores, quienes incluso se apoyaban técnicamente en sus hijos, sobrinos y amigos, para poder realizar las cápsulas radiales de contenido educativo.

5. En tanto la comunicación intercultural y el contexto donde se generan los mensajes se evidenció que la mayoría de los contenidos creados por profesores y estudiantes, demuestran en el discurso de las producciones radiofónicas una relación con la realidad en que viven inmersos ambos grupos de actores. Los mensajes se contextualizan y vinculan con temas como la cultura indígena, la salud, los problemas comunales, el territorio y la naturaleza (todas las escuelas comparten un marco geográfico de características exuberante) y el etnodesarrollo (Bonfil, 1982), a través de programas en vivo o cápsulas radiales educativas. Dichos mensajes generalmente se plantean desde dos perspectivas, una propia, particular e intimista, relacionada a elementos culturales del lugar, la comunidad y los habitantes y, por otro lado, los mensajes más universales, menos locales y generales, aquellos que se adhieren a la cultura hegemónica y sus códigos. La funcionalidad comunicativa podríamos aquí describirla como una hibridación de los contenidos y mensajes radiofónicos, los cuales ponen de manifiesto dos visiones de mundo (el local y el universal). Esto se une a una coherencia discursiva muy particular en tanto que, la mayoría de los programas y producciones de radio que revisamos y analizamos, en todas las escuelas que crearon y produjeron material, poseen una línea discursiva en la estructura de los mensajes, que se corresponde, directamente con la idea de hibridismo ya planteada. Las niñas y niños rescatan en sus guiones elementos de ese "limbo" situacional de estar entre dos realidades. 
6. Otro componente esencial dentro de la funcionalidad comunicativa es el referido a la pertinencia didáctica del mensaje. Todo el material radiofónico producido ostentó un carácter pedagógico desde dos perspectivas, la primera a partir del hecho per se de las actividades escolarizadas que demandan hacer radio trasnversalizando la enseñanza, la segunda desde el discurso y los mensajes de las producciones hechas por los estudiantes, donde se deja ver una línea pedagógica en las temáticas y la forma de abordar el conocimiento. Igualmente, en esta etapa se evidenciaron elementos en relación con la cultura, sobre todo en los aspectos lingüísticos referidos al aprendizaje del chesungun como segunda lengua después del castellano. Aquí entramos también en el campo de los códigos comunicativos y sus categorías, sea que el tipo de léxico utilizado por los estudiantes en la mayoría de los casos responde, para la producción de guiones, a palabras y términos utilizados en la cotidianidad, sin considerarse aquellos formalismos que pretende el presentarse ante los medios de comunicación. En tal sentido mucho del lenguaje utilizado es elemental, casi siempre en castellano, salvo algunas excepciones que hemos anteriormente descrito con respecto al chesungun, idioma representativo de la cultura mapuche-huilliche.

7. La idea de alfabetizar en lenguas indígenas es un tema recurrente hoy en día, sobre todo en las nuevas generaciones, quienes buscan honrar la herencia cultural de sus antepasados rescatando el idioma y las formas de comunicación ancestrales (Mosonyi, 2012). Uno de los elementos que observó este estudio, es que, desde la radio educativa, pueden tejerse iniciativas que potencien planes de revitalización cultural con foco en las lenguas de los pueblos originarios. La naturaleza del lenguaje radiofónico plasmado en la palabra, la música, los efectos y el silencio, puede fomentar didácticas que favorezcan el aprendizaje de idiomas ancestrales como el chesungun. Los estudiantes de las tres escuelas sienten un interés particular por hablar la lengua de sus ancestros, pero el sistema escolar, más allá de la educadora tradicional, no les ofrece herramientas para que ese aprendizaje sea sistemático y concreto. Ninguno de los niños que entrevistamos habla chesungun de manera fluida. Solo expresan algunos términos y frases breves.

8. La radio es un elemento dínamo que logra activar procesos no solo educativos desde una perspectiva estrictamente pedagógica, sino que también puede operativizar elementos exógenos tales como los representados en esta investigación, entiéndase la alfabetización mediática y la idea de una comunicación intercultural. A partir de los espacios que genera el "hacer radio", se evidenció que, en el entorno del proceso de producción, las relaciones entre estudiantes y profesores se potenciaron positivamente, en el entendido de que los docentes no son en su totalidad de origen indígena, como tampoco lo son en su totalidad los estudiantes. En el proceso comunicativo entre los diferentes actores, intra e infra escuelas, se evidenció la capacidad de comprender al otro y de compartir sus intereses, ya fueran estos de carácter educativo, sociales, culturales, etc. Desde las actividades para producir radio, se construyeron estadios de solidaridad y trabajo en equipo entre los estudiantes, profesores, directivos de los colegios y apoderados, los cuales determinaron espacios altamente dialógicos que dejan como evidencia niveles importantes de tolerancia y respeto hacia la diversidad cultural y la sana convivencia.

\section{Conclusiones}

A partir de los diferentes elementos que hemos evidenciado en el presente trabajo, podemos aseverar que la alfabetización mediática puede considerarse como una instancia útil para fortalecer y dinamizar los procesos comunicativos interculturales.

En ese sentido, se advierte la necesidad de generar instancias, particularmente críticas y reflexivas, desde el ámbito de la educomunicación (Orozco, 2014), que permitan llevar a la práctica acciones 
concretas en formato de planes y proyectos que concreticen una adecuada alfabetización en medios, por ejemplo, actividades sobre el uso de la radio educativa en el contexto escolar desde el marco de la educación intercultural bilingüe.

Por otro lado, al situarnos en escenarios conectados con las realidades y vida de los pueblos originarios, así como con comunidades indígenas o cualquier otro grupo con ciertas especificidades culturales, las propuestas cobran mayor relevancia pues se transforman en canal de comunicación con otras perspectivas de mundo, con otras culturas, con el "otro" (Esquirol, 2005), generando aquello que se denomina diálogo de saberes, lo cual genera espacios que exaltan y fomentan la diversidad cultural y una cultura de paz.

La radio sigue siendo un medio pertinente, aún en tiempos de comunicación digital (Peppino, 1999). La capacidad de cobertura, sobre todo en contextos de ruralidad, sigue siendo significativa y permite que sea un medio de comunicación aún accesible para muchos. En este sentido, los estudios y enfoques que sobre la radio se han realizado con respecto a su uso educativo, permiten que siga siendo un recurso recurrente para dinamizar los procesos de enseñanza y aprendizaje, así como la innovación educativa. En términos generales, podríamos aseverar que la radio desde su aplicación en contextos educativos en cualquiera de sus niveles formativos es una herramienta para impulsar planes de alfabetización mediática desde un punto de vista metadisciplinar (Pérez-Maíllo, Sánchez-Serrano y Pedrero-Esteban, 2018), además de potenciar estrategias de aprendizaje activo (Jerez, 2015), que redundarán positivamente en la formación de los estudiantes.

Una nueva ventana y posibilidad que se le abre a la radio educativa es la posibilidad de estar al servicio de la formación en valores, aquella relacionada con las habilidades blandas. La producción radial requiere competencias para el trabajo en equipo y el juego de roles, lo que permitiría configurar instancias que podrían replicarse de igual manera en otras situaciones de la vida, elemento evidenciado en esta investigación en las interacciones entre los estudiantes y los docentes a la hora de producir contenidos radiofónicos.

Por otro lado, la radio educativa de hoy debe ser entendida desde nuevas modalidades que trasciendan las históricas transmisiones mediante ondas hertzianas. En nuestros días, la radio en general se sitúa en infinidad de espacios y bajo distintas características, apoyándose en plataformas que permiten la emisión del mensaje de manera síncrona o asíncrona, siendo esto una circunstancia que la coloca en un lugar privilegiado dentro de la gama de multimedios que existen. Desde el punto de vista educativo, los mismos elementos descritos anteriormente fortalecen su función pues desde los distintos formatos (cápsulas, documentales, noticieros, narraciones, historias, radioteatro, etc.) es posible reutilizar los contenidos e incluso, editarlos para adecuarlos si fuera necesario. En definitiva, la radio ha vivido, al menos en los últimos 10 años, un proceso considerable de reinvención a partir de quien parecía venían a extinguirla; los medios digitales.

Finalmente, el valor de las perspectivas e interfaces aquí propuestas y analizadas, cobran significado en tanto sus interacciones, así, la comunicación intercultural genera esferas significativamente importantes para equilibrar las asimetrías socio-técnico-comunicativas que existen en nuestras sociedades multiculturales (Sagástegui, 2005), siendo que los ecosistemas mediáticos actuales (McLuhan, 2003), producen un exceso de interactividad, lo que ha impulsado desequilibrios importantes en el acceso a la información pero sobre todo, ha modificado la manera de comunicarnos. De ahí la necesidad de la alfabetización mediática para potenciar las habilidades comunicativas en medio de lo que se conoce como infoxicación mediática (Aguaded, Pérez Escoda y Peñalva, 2018), aspecto para lo cual no están preparados los sectores más vulnerables de la sociedad, significando esto, una tarea que requiere cierta prisa, pues aún hoy sigue estando pendiente. 


\section{Bibliografía}

Abdallah-Pretceille, M. (2001). La educación intercultural. Idea Books.

Aguaded, I., Marín-Gutiérrez I. y Díaz-Parejo, E. (2018). Desarrollo de la competencia mediática en el contexto Ibero-americano. Revista Letral, no 20, 156-182. https://doi.org/10.30827/RL

Aguaded, I., Pérez Escoda A., \& Peñalva, S. (2018). Integrados comunicacionales: Competencias, TIC y convergencia digital en la transformación de competencias mediáticas. En Aguaded y Romero Rodríguez (Eds.). Competencias mediáticas en medios digitales emergentes. Comunicación Social Ediciones y Publicaciones.

Alsina, M. R. (1999). La comunicación Intercultural. Anthropos.

Araya-Rivera, C. (2017). La radio estudiantil como estrategia didáctica innovadora. Actualidades investigativas en educación. 17(3), 1-32. https://doi.org/10.15517/AIE.V17I3.30098

Arteaga, C. Et Al. (2004). La radio como medio para la educación. Razón y Palabra, (36). https:// bit.ly/3AIHAtd

Bonfil, G. (1982). El Etnodesarrollo: Sus Premisas Jurídicas, Políticas, y de Organización. Francisco Rojas América Latina; Etnodesarrollo, Etnocidio, 180-205. Flacso.

Braslavsky, B. (2013). Enseñar a entender lo que se lee. La alfabetización en la familia y en la escuela. Fondo de Cultura Económica.

Buckingham, D. (2003). Educación en medios. Alfabetización, aprendizaje y cultura contemporánea. Paidós.

Buckingham, D. (2019). Teaching media in a 'post-truth' age: fake news, media bias and the challenge for media/digital literacy education. Culture and Education, 31 (2), 213-231, https://doi. org/10.1080/11356405.2019.1603814

Buitrago, A., García, A. y Gutiérrez, A. (2016). Perspectiva histórica y clave actuales de la diversidad terminológica aplicada a la educación mediática. edmetic, 6(2), 81-104. https://doi. org/10.21071/edmetic.v6i2.7002

Carias Pérez, F. (2018). Los medios audiovisuales en la propuesta pedagógica intercultural de la Universidad Nacional Experimental Indígena del Tauca. En Huertas, A. y Bailén, M. (Ed.).

Culturas indígenas: investigación, comunicación y resistencia (pp. 169-180). InCom-UAB Publicacions. https://ddd.uab.cat/pub/llibres/2018/189425/16eBook_incomuabindigenes 16.pdf

Carias Pérez, F., Hernando Gómez, Á., \& Marín-Gutiérrez, I. (2021). Uso educativo de la radio en tiempos de pandemia en escuelas rurales chilenas. Chasqui. Revista Latinoamericana de Comunicación, 1(146), 59-76. https://doi.org/10.16921/chasqui.v1i146.4381

Castillo Villalobos, M. L., Monroy Ordóñez, M. C. D. P., \& Tíjaro Corredor, Y. L. (2021). Podcast and school radio: a reflection of a practice developed in Neusa - Cundinamarca. Revista Iberoamericana de la educación, 4(1), 31-52. https://bit.ly/3xJYC8s

Consani, M. (2018). Como usar o radio na sala de aula. Editora Contexto.

Escoffier, J. (1991). The Limits of Multiculturalism, Socialist Review, 3-4. 61-73.

Escuder, S. (2019). Regionalización de la brecha digital. Desarrollo de la infraestructura de las TIC en Latinoamérica y Uruguay. Paakat: Revista de Tecnología y Sociedad, 17 (9), 1-26. http:// dx.doi.org/10.32870/Pk.a9n17.356 
Esquirol, J. M. (2005). Uno mismo y los otros. De las experiencias existenciales a la interculturalidad. Hender.

Ferrés, J. (2007). La competencia en comunicación audiovisual: Dimensiones e indicadores. Comunicar, 15(29),100-107. https://doi.org/10.3916/C29-2007-14

Ferrés, J. y Piscitelli, A. (2012). La competencia mediática: propuesta articulada de dimensiones e indicadores. Comunicar, (38), 75-82. http://dx.doi.org/10.3916/C38-2012-02-08

García Canclini, N. (2004). Diferentes, Desiguales y Desconectados. Mapas de la interculturalidad. Gedisa.

González Castro, C. (2014). Metáforas de un conflicto. El discurso étnico chileno-mapuche de la modernidad. Alquimia ediciones.

González Conde, M.J. (2014). La ciberradio como modalidad interactiva de comunicación multimedia, en su dimensión expresiva hipertextual e hipermedial, en Contenidos innovadores en la universidad actual, Mc Graw Hill Educación, 363-379.

Grise, P.J., Epstein, K.L. \& Lukin, T.G. (1974). Educational Radio: A Review of the Literature. Florida State University, Tallahassee.

Gutiérrez Porlan, Ma . \& Rodríguez Cifuentes, T. (2010). Haciendo historia del podcats. Referencias sobre su origen y evolución. En I.M. Solano (Ed.), Podcast Educativo. Aplicaciones y orientaciones del M-Learning para la enseñanza (pp. 37-54). MAD Editorial.

Hadriel, T. y Cogo, D. (2020). (In)visibilidades sociocomunicacionais na diáspora queer. Palabra Clave, 23(3), e2332. https://doi.org/10.5294/pacla.2020.23.3.2 https://doi.org/10.5294/ pacla.2020.23.3.2

Jerez, O. (2015). Aprendizaje activo, diversidad e inclusión. Enfoques, metodología y recomendaciones para su implementación. Ediciones Universidad de Chile.

Kaplún, M. (1998). Una pedagogía de la comunicación. Ediciones de la Torre.

Labio-Bernal, A., Romero-Domínguez, L.R., García Orta, M.J. y García-Prieto, V. (2020). Competencia digital informacional, alfabetización mediática y periodismo. Un análisis de caso a través del proyecto “La Prensa en las escuelas". Icono, 18 (2), 58-83. https://doi.org/10.7195/ri14.v18i1.1387

Leiva Olivencia, J. J. y Márquez Pérez, M. (2012). La comunicación intercultural: una herramienta de inclusión en los contextos educativos de diversidad cultural. Revista de Pedagogía, 33 (93), 71-93. https://bit.ly/33DyaAL

Martínez, A. \& Sánchez Vera, M. (2010). Aspectos técnicos y legales del diseño de podcats. Herramientas, aplicaciones, servicios y licencias. En I.M. Solano (Ed.), Podcast Educativo. Aplicaciones y orientaciones del M-Learning para la enseñanza (pp. 75-88). MAD Editorial.

McLuhan, M. (2003). Understanding Media. Ginko Press.

McMillan, J., \& Schumacher, S. (2005). Investigación educativa. Pearson.

Medrano, C., cuervo, S. y Martínez, J. (2019). Programa para favorecer las competencias mediáticas e informacionales de los adolescentes. Pirámide.

Méndiz Rojas, H. (2004). La radio va a la escuela: articulación entre comunicación y educación. Comunicar, (22), 115-120. https://doi.org/10.3916/C22-2004-17 
Merayo, A. (2000). Identidad, sentido y uso de la radio educativa. Memorias III Congreso Internacional Cultura y Medios de Comunicación. Salamanca: Universidad Pontificia de Salamanca, 387-404. https://bit.ly/3fXiYF9

Moreno-Correa, S. (2020). La innovación educativa en los tiempos del Coronavirus. Salutem Scientia Spiritus, 6 (1), 14-26. https://bit.ly/3uKglft

Mosonyi, E.E. (2012). El discurso sobre la irreversible extinción de las lenguas: Un atentado contra la interculturalidad. Boletín de lingüística. 24 (37-38), 197-215. https://bit.ly/3uKRGHr

Muñoz, J. (1994). Radio Educativa. Cervantes.

Orozco, G (2014). Educomunicação: recepção mediática, aprendizagens e cidadania. Paulinas.

Pareja de Vicente, D., Leiva Olivencia, J.J. \& Matas Terrón, A. (2020). Percepciones sobre diversidad cultural y comunicación intercultural de futuros maestros. Revista Electrónica Interuniversitaria de Formación del Profesorado, 23(1), 75-87. https://doi.org/10.6018/reifop.403331

Peppino, A.M. (1999). Radio Educativa, popular y comunitaria en América Latina. Plaza y Valdés.

Pereira, E. S. (2010) Ciborgues indigen@s.br: a presença nativa no ciberespaço, Difusão.

Pérez Escoda, A. (2017). Alfabetización mediática, TIC y competencias digitales. UOC.

Pérez Tornero, J.M. (2015). La emergencia de la alfabetización mediática e informacional: un nuevo paradigma para las políticas públicas y la investigación. Revista Telos, (100), 99-102. https://bit. ly/2RRA8uZ

Pérez Tornero, J.M. y Varis, T. (2012). Alfabetización mediática y nuevo humanismo. UOC.

Pérez Tornero, J. M. y Tayie, S. (2012). La formación de profesores en educación en medios: Currículo y experiencias internacionales. Comunicar, 20(39), 10-14. https://doi.org/10.3916/C39-2012$\underline{02-00}$

Pérez-Maíllo, A., Sánchez-Serrano, C., \& Pedrero-Esteban, L. M. (2018). Viaje al Centro de la Radio. Diseño de una experiencia de alfabetización transmedia para promover la cultura radiofónica entre los jóvenes. Comunicación y Sociedad, (33), 171-201. https://doi.org/10.32870/cys. $\underline{\mathrm{v} 0 \mathrm{i} 33.7031}$

Perona, J. \& Veloso, M. (2007). Modalidades educativas de la radio en la era digital. Icono 14 (9), 12-37. https://bit.ly/2VLUmYW

Rodero-Antón, E. (2008). Educar a través de la radio. Signo y Pensamiento, 27(52), 97-109. https:// bit.ly/3jPw34N

Sabariego, M., Dorio, I., \& Massot, I. (2019). Metodología de la investigación educativa. En R. Bisquerra (Ed.), Metodología de la investigación educativa (pp. 329-366). La Muralla.

Sagástegui, D. (2005). La apropiación social de la tecnología. Un enfoque sociocultural del conocimiento. Universidad de Guadalajara.

Sevillano García, M. L. (2011). Medios, recursos didácticos y tecnología educativa. Pearson Educación.

Soep, E. (2012). Generación y recreación de contenidos digitales por los jóvenes: implicaciones para la alfabetización mediática. Comunicar, 38 (19), 93-100. https://doi.org/10.3916/C38-201202-10 
Toboso Martín, M. (2013). Entre el uso y el no uso de la tecnología: un enfoque discursivo de la apropiación tecnológica. Intersticios. Revista Sociológica de Pensamiento Crítico, 7 (2), 201-214. https://bit.ly/3bkUBPp

Tuhiwai Smith, L. (2016). A descolonizar las metodologías. Investigación y pueblos indígenas. LOM.

UNESCO (2011). Alfabetización Mediática e Informacional (AMI), curriculum para profesores. UNESCO. 